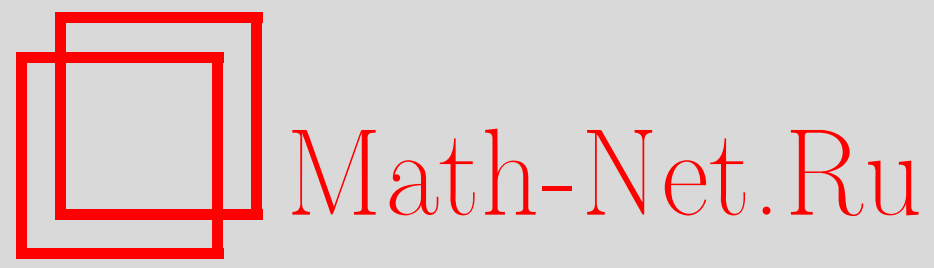

В. Г. Овчинников, Алгоритм типа покоординатного спуска на обладающем свойством усиленной замены множестве точек координатной решетки, Вестн. Сам. гос. техн. ун-та. Сер. Физ.-мат. науки, 2004, выпуск 27, 14-19

DOI: https://doi.org/10.14498/vsgtu260

Использование Общероссийского математического портала Math-Net.Ru подразумевает, что вы прочитали и согласны с пользовательским соглашением

http://www.mathnet.ru/rus/agreement

Параметры загрузки:

IP : 54.162 .85 .209

26 апреля 2023 г., $16: 17: 44$ 


\title{
Методы оптимизации
}

\author{
УДК 519.3
}

\section{В.Г. Овчинников}

\section{АЛГОРИТМ ТИПА ПОКООРДИНАТНОГО СПУСКА НА ОБЛАДАЮЩЕМ СВОЙСТВОМ УСИЛЕННОЙ ЗАМЕНЫ МНОЖЕСТВЕ ТОЧЕК КООРДИНАТНОЙ РЕШЕТКИ}

\begin{abstract}
Для задачи минимизаџии сепарабельной координатно-выпуклой функции на конечном и обладающем свойством усиленной замены множестве точек координатной решетки предлагается и обосновьвается алгоритм типа покоординатного спуска.
\end{abstract}

Введение. В настоящей работе продолжены исследования работ [1-4]. Для нахождения решения основной задачи (задачи минимизации сепарабельной координатно-выпуклой функции на конечном и обладающем свойством усиленной замены множестве точек координатной решетки) рассматривается семейство вспомогательных задач, устанавливаются лемма о связи между оптимальными решениями этих задач (аналог леммы 1 из работы[5]), лемма о соотношениях между их оптимальными значениями (аналог леммы 2 той же работы) и с помощью этих лемм показывается, как эффективный (в терминологии для матроидов [6] - «жадный») алгоритм покоординатного спуска и подход к его обоснованию для задачи [5] используются в их некоторой модификации для основной задачи. [1-4].

Определения. В дальнейшем будут использоваться обозначения и терминология работ

Под координатной решеткой $(\Gamma, \prec)$ понимается множество $\left\{\left(x_{1}, \ldots, x_{n}\right): x_{i} \in \Gamma_{i}, \forall i \in N\right\}$ с отношением порядка, определяемым условием: $\left(x_{1}, \ldots, x_{n}\right) \prec\left(y_{1}, \ldots, y_{n}\right)$ тогда и только тогда, когда $x_{i} \prec_{i} y_{i}, \forall i \in N=\{1,2, \ldots, n\}$, где $\Gamma_{i}$ - цепь с отношением порядка $\prec_{i}$, в которой конечны все интервалы $\left[a_{i}, b_{i}\right]=\left\{x_{i} \in \Gamma_{i}: a_{i} \prec_{i} x_{i} \prec_{i} b_{i}\right\}$, т.е. $\left|\left[a_{i}, b_{i}\right]\right|<\infty$. Следовательно, для всякой точки $x=\left(x_{1}, \ldots, x_{n}\right)$ решетки и любого индекса $j \in N$, если элемент $x_{j}$ не минимален, существует единственная точка $\tau_{j}(x)=\left(x_{1}^{\tau_{j}}, \ldots, x_{n}^{\tau_{j}}\right)$, где $x_{i}^{\tau_{j}}=x_{i}$ при $i \neq j$ и $x_{j}^{\tau_{j}}$ - элемент, непосредственно предшествующий $x_{j}$ в цепи $\Gamma_{j}$, а если $x_{j}$ не максимален - существует единственная точка $\pi_{j}(x)=\left(x_{1}^{\pi_{j}}, \ldots, x_{n}^{\pi_{j}}\right)$, где $x_{i}^{\pi_{j}}=x_{i}$ при $i \neq j$ и $x_{j}{ }^{\pi_{j}}$ - элемент, непосредственно следующий за $x_{j}$ в цепи $\Gamma_{j}$

Сепарабельной координатно-выпуклой функцией (СКВФ) называем заданную на множестве точек решетки $(\Gamma, \prec)$ функцию $f(x)=\sum_{i=1}^{n} f_{i}\left(x_{i}\right)$, для которой всякий ее $i$ - градиент $f\left(\pi_{i}(x)\right)-f(x)$ не убывает при переходе от $x$ к точке $\pi_{i}(x)$.

Лемма 1. Для СКВФ $f(x)$ выполняются следующие условия:

$$
\left(w_{j} \neq v_{j} \prec_{j} w_{j}\right) \Rightarrow f_{j}\left(v_{j}^{\pi_{j}}\right)-f_{j}\left(v_{j}\right) \leq f_{j}\left(w_{j}\right)-f_{j}\left(w_{j}^{\tau_{j}}\right), \quad \forall v_{j}, w_{j} \in \Gamma_{j}, \quad \forall j \in N .
$$

Доказательство. Из условий $v_{j}, w_{j} \in \Gamma_{j}, w_{j} \neq v_{j} \prec_{j} w_{j}$ следует, что $v_{j}$ не максимален в $\Gamma_{j}$ и $v_{j}^{\pi_{j}} \in \Gamma_{j}, \quad w_{j} \quad$ не минимален в $\Gamma_{j}$ и $w_{j}^{\tau_{j}} \in \Gamma_{j}, \quad\left|\left[v_{j}, w_{j}\right]\right| \geq 2$.

При $\left|\left[v_{j}, w_{j}\right]\right|=2$ из определения $v_{j}^{\pi_{j}}$ имеем $v_{j}^{\pi_{j}} \neq v_{j} \prec_{j} v_{j}^{\pi_{j}} \prec_{j} w_{j} \quad$ и поэтому $v_{j}^{\pi_{j}}=w_{j}$, а из определения $w_{j}{ }^{\tau_{j}}$ имеем $v_{j} \prec_{j} w_{j}{ }^{\tau_{j}} \prec_{j} w_{j} \neq w_{j}{ }^{\tau_{j}}$ и потому $w_{j}{ }^{\tau_{j}}=v_{j}$. Отсюда следует, что при $k=1$ установлена справедливость следующего предложения. 
Предложение $T(k)$. Для СКВФ $f(x)$ выполняются следующие условия:

$$
\left(v_{j} \prec_{j} w_{j}\right) \wedge\left(\left[v_{j}, w_{j}\right] \mid=k+1\right) \Rightarrow f_{j}\left(v_{j}^{\pi_{j}}\right)-f_{j}\left(v_{j}\right) \leq f_{j}\left(w_{j}\right)-f_{j}\left(w_{j}^{\tau_{j}}\right), \quad \forall v_{j}, w_{j} \in \Gamma_{j}, \quad \forall j \in N .
$$

Выбирая натуральное $m$, для которого справедливо $T(m)$, азатем полагая $k=m+1$ и выбирая по произволу индекс $j \in N$ и элементы $x_{j}, y_{j} \in \Gamma_{j}$ из условий $x_{j} \prec_{j} y_{j},\left|\left[x_{j}, y_{j}\right]\right|=k+1=m+2$, покажем, что $f_{j}\left(x_{j}^{\pi_{j}}\right)-f_{j}\left(x_{j}\right) \leq f_{j}\left(y_{j}\right)-f_{j}\left(y_{j}{ }^{\tau_{j}}\right)$, т.е. установим справедливость предложения $T(m+1)$ и, таким образом, по индукции, получим лемму.

Из условий выбора $x_{j}, y_{j} \in \Gamma_{j}$, т.е. из соотношений $x_{j} \prec_{j} y_{j},\left|\left[x_{j}, y_{j}\right]\right|=m+2$, следует, что $x_{j} \neq y_{j}, \quad x_{j}^{\pi_{j}} \in \Gamma_{j}, \quad x_{j}^{\pi_{j}} \neq x_{j} \prec_{j} x_{j}^{\pi_{j}} \prec_{j} y_{j},\left|\left[x_{j}, y_{j}\right]\right|=1+\left|\left[x_{j}^{\pi_{j}}, y_{j}\right]\right|,\left|\left[x_{j}^{\pi_{j}}, y_{j}\right]\right|=m+1$.

Положим $\mathrm{z}_{\mathrm{j}}=x_{j}^{\pi_{j}}$. Из предыдущего абзаца имеем $\mathrm{z}_{\mathrm{j}} \prec_{j} y_{j}, \quad\left|\left[\mathrm{z}_{\mathrm{j}}, y_{j}\right]\right|=m+1, \mathrm{z}_{\mathrm{j}} \in \Gamma_{j}$. Отсюда с помощью предложения $T(m)$, справедливого по выбору $m$, получаем

$$
f_{j}\left(z_{j}^{\pi_{j}}\right)-f_{j}\left(z_{j}\right) \leq f_{j}\left(y_{j}\right)-f_{j}\left(y_{j}^{\tau_{j}}\right) .
$$

Из равенства $\mathrm{z}_{\mathrm{j}}=x_{j}{ }^{\pi_{j}}$ и определения $f(x)$ имеем

$$
f_{j}\left(x_{j}^{\pi_{j}}\right)-f_{j}\left(x_{j}\right) \leq f_{j}\left(z_{j}^{\pi_{j}}\right)-f_{j}\left(z_{j}\right) .
$$

Отсюда и из предыдущего неравенства следует требуемое неравенство. Лемма доказана.

Аналогично проверяется и следующая лемма.

Лемма 2. Если точки $v, w$ решетки $(\Gamma, \prec)$ и индексы $j, k$ удовлетворяют включениям $j \in I(v, w), k \in I(w, v)$, то $j \neq k$ и равенства $x_{j}=v_{j}{ }^{\pi_{j}}, x_{k}=v_{k}{ }^{\tau_{k}}, x_{s}=v_{s}, \forall s \in N \backslash\{j, k\}$, покоординатно задают такую точку $x$ решетки $(\Gamma, \prec)$, что $x=\pi_{j}\left(\tau_{k}(v)\right)$.

Свойство усиленной замены. Для множества $Q$ точек решетки $(\Gamma, \prec)$ следующее свойство замены - это усиленный вариант известной аксиомы Штейница о замене (см. 6.10 (ii) [7]):

для любой пары $x, y$ различных точек множества $Q$ и всякого индекса $j \in I(x, y)=\left\{i \in N: x_{i} \prec_{i} y_{i}, x_{i} \neq y_{i}\right\}$ найдется такой индекс $k \in I(y, x)$, что $\pi_{j}\left(\tau_{k}(x)\right) \in Q$ и $\pi_{k}\left(\tau_{j}(y)\right) \in Q$.

В случае, когда решетку $(\Gamma, \prec)$ образуют булевы $n$ - векторы, этим свойством обладает множество $Q$ характеристических векторов баз в матроиде $M(N)$ (ср. с упражнением VI.1.9 на странице 312 [7]).

Задача $\boldsymbol{A}$. Основной задачей настоящей статьи является следующая задача.

При предположениях, что $f(x)$ есть СКВФ, а множество $Q$ точек решетки $(\Gamma, \prec)$ является конечным и обладает свойством усиленной замены, требуется минимизировать $f(x)$ на множестве Q, т.е. найти такую точку $x \in Q$, что $f(x)=\min \{f(y): y \in Q\}$.

Для нахождения решения этой задачи рассмотрим семейство вспомогательных задач следующего вида.

Задача $\boldsymbol{A}_{m}{ }^{\boldsymbol{x}_{L}}\left(\sigma_{m}\right)$. Для индекса $m$ собственного подмножества $L \subset N \backslash\{m\}$, точки $x$ множества $Q$ и параметра $\sigma_{m} \in P_{m}^{x_{L}}=\left\{y_{m}: y=\left(y_{1}, \ldots, y_{n}\right) \in Q, i \in L \Rightarrow y_{i}=x_{i}, \forall i \in N\right\}$ названная вспомогательная задача формулируется следующим образом.

При предположениях задачи $\boldsymbol{A}$ требуется минимизировать $f(x)$ на множестве $Q_{m}{ }^{x_{L}}\left(\sigma_{m}\right)=\left\{\left(y_{1}, \ldots, y_{n}\right) \in Q: y_{m}=\sigma_{m}, i \in L \Rightarrow y_{i}=x_{i}, \forall i \in N\right\}, \quad$ т.е. $\quad$ найти $\quad$ такую $\quad$ точку $w \in Q_{m}{ }^{x_{L}}\left(\sigma_{m}\right)$, что $f(w)=F_{m}{ }^{x_{L}}\left(\sigma_{m}\right)$, где $F_{m}{ }^{x_{L}}\left(\sigma_{m}\right)=\min \left\{f(y): y \in Q_{m}{ }^{x_{L}}\left(\sigma_{m}\right)\right\}$.

Лемма 3 (ср. с леммой 1 [5]). Если для функции $f(x)$ и множества $Q$ точек решетки $(\Gamma, \prec)$ выполняются предположения задачи $\boldsymbol{A}, x$ - точка множества $Q$, а индекс $m \in N$ и подмножество индексов $L$ удовлетворяют строгому включению $L \subset N \backslash\{m\}$, то справедливы следующие предложения о связи между решениями вспомогательных задач: 
1. Если параметр $\sigma_{m} \in P_{m}{ }^{x_{L}}$ и точка $w \in Q_{m}{ }^{x_{L}}\left(\sigma_{m}\right)$ удовлетворяют равенст-

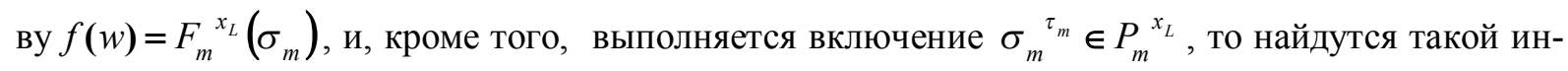
декс $j \in N$ и такая точка $v \in Q$, что

$v \in Q_{m}{ }^{x_{L}}\left(\sigma_{m}{ }^{\tau_{m}}\right), f(v)=F_{m}{ }^{x_{L}}\left(\sigma_{m}{ }^{\tau_{m}}\right), v_{j}=w_{j}^{\pi_{j}}, v_{m}=w_{m}{ }^{\tau_{m}}, v_{s}=w_{s}, \forall s \in N \backslash\{j, m\}, m \neq j \notin L$.

2. Если параметр $\sigma_{m} \in P_{m}{ }^{x_{L}}$ и точка $w \in Q_{m}{ }^{x_{L}}\left(\sigma_{m}\right)$ удовлетворяют равенству $f(w)=F_{m}{ }^{x_{L}}\left(\sigma_{m}\right)$, и, кроме того, выполняется включение $\sigma_{m}{ }^{\pi_{m}} \in P_{m}^{x_{L}}$, то найдутся такой индекс $j \in N$ и такая точка $v \in Q$, что

$v \in Q_{m}{ }^{x_{L}}\left(\sigma_{m}{ }^{\pi_{m}}\right), f(v)=F_{m}{ }^{x_{L}}\left(\sigma_{m}{ }^{\pi_{m}}\right), v_{j}=w_{j}^{\tau_{j}}, v_{m}=w_{m}{ }^{\pi_{m}}, v_{s}=w_{s}, \forall s \in N \backslash\{j, m\}, m \neq j \notin L$.

Доказательство. При условиях леммы докажем, что справедливо предложение 1 (справедливость предложения 2 доказывается аналогично).

Из условий леммы имеем $w \in Q_{m}^{x_{L}}\left(\sigma_{m}\right), f(w)=F_{m}{ }^{x_{L}}\left(\sigma_{m}\right), \sigma_{m}^{{ }^{\tau_{m}}} \in P_{m}^{x_{L}}$. Из последнего включения следует, что множество $Q_{m}{ }^{x_{L}}\left(\sigma_{m}{ }^{\tau_{m}}\right)$ является непустым. Из этого множества, как подмножества конечного множества $Q$, выберем такую точку $u \in Q_{m}{ }^{{ }^{x}}\left(\sigma_{m}{ }^{\tau_{m}}\right)$, что $f(u)=F_{m}{ }^{x_{L}}\left(\sigma_{m} \tau_{m}\right)$.

Из включений $u \in Q_{m}{ }^{{ }_{L}}\left(\sigma_{m}{ }^{\tau_{m}}\right), w \in Q_{m}{ }^{x_{L}}\left(\sigma_{m}\right)$ имеем $u \in Q, \quad w \in Q, u_{m}=\sigma_{m}{ }^{\tau_{m}}, w_{m}=\sigma_{m}$. Отсюда (по определению $\sigma_{m}^{\tau_{m}}$ ) получаем $u_{m} \prec_{m} w_{m} \neq u_{m}, m \in I(u, w), u \neq w$. Так как по условиям леммы множество $Q$ обладает свойством усиленной замены, то для различных точек $u, w$ множества $Q$ по индексу $m \in I(u, w)$ найдется такой индекс $j \in I(w, u)$, что $\pi_{m}\left(\tau_{j}(u)\right) \in Q$, $\pi_{j}\left(\tau_{m}(w)\right) \in Q$. Заметим, что из включений $u \in Q_{m}{ }^{x_{L}}\left(\sigma_{m}{ }^{\tau_{m}}\right), w \in Q_{m}{ }^{x_{L}}\left(\sigma_{m}\right)$ следует также, что $j, m \notin L$. Полагая здесь $v=\pi_{j}\left(\tau_{m}(w)\right), y=\pi_{m}\left(\tau_{j}(u)\right)$, с помощью леммы 2 находим:

$$
v_{j}=w_{j}^{\pi_{j}}, \quad v_{m}=w_{m}^{\tau_{m}}, v_{s}=w_{s}, y_{j}=u_{j}^{\tau_{j}}, \quad y_{m}=u_{m}^{\pi_{m}}, y_{s}=u_{s}, \forall s \in N \backslash\{j, m\}, m \neq j \notin L .
$$

Из (1) с помощью равенств $u_{m}=\sigma_{m}{ }^{\tau_{m}}, w_{m}=\sigma_{m}$ (имеющихся по выбору $u$ и $w$ ), равенства $u_{m}{ }^{\pi_{m}}=\sigma_{m}$, вытекающего из них, как при доказательстве леммы 1 , получаем

$$
v_{j}=w_{j}^{\pi_{j}}, y_{j}=u_{j}{ }^{\tau_{j}}, v_{m}=\sigma_{m}{ }^{\tau_{m}}=u_{m}, y_{m}=\sigma_{m}=w_{m}, v_{s}=w_{s}, \quad y_{s}=u_{s}, \forall s \in N \backslash\{j, m\}, m \neq j \notin L
$$

Поэтому $v \in Q_{m}{ }^{x_{L}}\left(\sigma_{m}{ }^{\tau_{m}}\right), y \in Q_{m}{ }^{x_{L}}\left(\sigma_{m}\right)$. Отсюда непосредственно, т.е. с помощью определений $F_{m}{ }^{x_{L}}\left(\sigma_{m}{ }^{\tau_{m}}\right)$ и $F_{m}{ }^{x_{L}}\left(\sigma_{m}\right)$, следует $f(v) \geq F_{m}{ }^{x_{L}}\left(\sigma_{m}{ }^{\tau_{m}}\right), f(y) \geq F_{m}{ }^{x_{L}}\left(\sigma_{m}\right)$, и, таким образом, с учетом выбора точек $u$ и $w$ имеем

$$
f(v) \geq F_{m}{ }^{x_{L}}\left(\sigma_{m}{ }^{\tau_{m}}\right)=f(u), \quad f(y) \geq F_{m}{ }^{x_{L}}\left(\sigma_{m}\right)=f(w) .
$$

Отсюда и из (1) следует, что для доказательства леммы достаточно получить неравенство

$$
f(v) \leq f(u) \text {. }
$$

По выбору функции $f(x)$, используя (2), получаем

$$
f(v)=f(w)+f_{j}\left(v_{j}\right)-f_{j}\left(w_{j}\right)+f_{m}\left(v_{m}\right)-f_{m}\left(w_{m}\right)=f(w)+f_{j}\left(w_{j} \pi_{j}\right)-f_{j}\left(w_{j}\right)+f_{m}\left(\sigma_{m}{ }^{\tau_{m}}\right)-f_{m}\left(\sigma_{m}\right) .
$$

Отсюда, используя неравенство $f(w) \leq f(y)$, имеющееся в (3), с помощью (2) получаем

$$
\begin{aligned}
f(v) & \leq f(y)+f_{j}\left(w_{j}^{\pi_{j}}\right)-f_{j}\left(w_{j}\right)+f_{m}\left(\sigma_{m} \tau_{m}\right)-f_{m}\left(\sigma_{m}\right)=f(u)+f_{j}\left(y_{j}\right)-f_{j}\left(u_{j}\right)+f_{m}\left(y_{m}\right)-f_{m}\left(u_{m}\right)+ \\
& +f_{j}\left(w_{j}^{\pi_{j}}\right)-f_{j}\left(w_{j}\right)+f_{m}\left(\sigma_{m}^{\tau_{m}}\right)-f_{m}\left(\sigma_{m}\right)=f(u)+f_{j}\left(u_{j}^{\tau_{j}}\right)-f_{j}\left(u_{j}\right)+f_{m}\left(\sigma_{m}\right)-f_{m}\left(\sigma_{m}{ }^{\tau_{m}}\right)+ \\
& +f_{j}\left(w_{j}^{\pi_{j}}\right)-f_{j}\left(w_{j}\right)+f_{m}\left(\sigma_{m}^{\tau_{m}}\right)-f_{m}\left(\sigma_{m}\right)=f(u)+f_{j}\left(u_{j}^{\tau_{j}}\right)-f_{j}\left(u_{j}\right)+f_{j}\left(w_{j}{ }_{j}\right)-f_{j}\left(w_{j}\right) .
\end{aligned}
$$

Таким образом, имеем

$$
f(v) \leq f(u)+f_{j}\left(u_{j}^{\tau_{j}}\right)-f_{j}\left(u_{j}\right)+f_{j}\left(w_{j}^{\pi_{j}}\right)-f_{j}\left(w_{j}\right) .
$$

Замечая теперь, что индекс $j$ выбирался из включения $j \in I(w, u)$, т.е. условий $w_{j} \prec_{j} u_{j} \neq w_{j}$, 
с помощью леммы 1 получаем $f_{j}\left(w_{j}^{\pi_{j}}\right)-f_{j}\left(w_{j}\right) \leq f_{j}\left(u_{j}\right)-f_{j}\left(u_{j}^{\tau_{j}}\right)$. Отсюда и из предыдущего неравенства следует требуемое неравенство (4). Лемма доказана.

Лемма 4 (ср. с леммой 2 [5]). Если для функции $f(x)$ и множества $Q$ точек решетки $(\Gamma, \prec)$ выполняются предположения задачи $\boldsymbol{A}, \boldsymbol{x}$ - точка множества $Q$, а индекс $m$ и подмножество индексов $L$ удовлетворяют строгому включению $L \subset N \backslash\{m\}$, то $F_{m}{ }^{{ }_{L}}\left(\sigma_{m}\right)$ является выпуклой функцией параметра $\sigma_{m} \in P_{m}{ }^{x_{L}}$, т.е.

$\left(\theta_{m} \prec_{m} \omega_{m} \neq \theta_{m}\right) \wedge\left(\left[\theta_{m}, \omega_{m}\right] \subseteq P_{m}^{x_{L}}\right) \Rightarrow F_{m}{ }^{x_{L}}\left(\theta_{m}{ }^{\pi_{m}}\right)-F_{m}{ }^{x_{L}}\left(\theta_{m}\right) \leq F_{m}{ }^{x_{L}}\left(\omega_{m}\right)-F_{m}{ }^{x_{L}}\left(\omega_{m}{ }^{\tau_{m}}\right), \forall \theta_{m}, \omega_{m} \in \Gamma_{m}$.

Доказательство. Для доказательства леммы достаточно убедиться, что разность $F_{m}{ }^{x_{L}}\left(\theta_{m}{ }^{\pi_{m}}\right)-F_{m}{ }^{x_{L}}\left(\theta_{m}\right)$ не убывает при переходе от параметра $\theta_{m} \in P_{m}^{{ }^{x_{L}}}$ К параметру $\theta_{m}{ }^{\pi_{m}} \in P_{m}{ }^{x_{L}}$ (поскольку с помощью этого свойства заключение леммы получается аналогично доказательству леммы 1).

Полагая $\sigma_{m}=\theta_{m}{ }^{\pi_{m}}$ и, следовательно, получая $\theta_{m}=\sigma_{m}{ }^{\tau_{m}}$, выразим указанное свойство с помощью неравенства $F_{m}{ }^{x_{L}}\left(\sigma_{m}\right)-F_{m}{ }^{x_{L}}\left(\sigma_{m}^{\tau_{m}}\right) \leq F_{m}{ }^{x_{L}}\left(\sigma_{m}^{{ }^{\pi_{m}}}\right)-F_{m}{ }^{x_{L}}\left(\sigma_{m}\right)$ или равносильного ему неравенства $F_{m}{ }^{x_{L}}\left(\sigma_{m}{ }^{\pi_{m}}\right)-2 F_{m}{ }^{x_{L}}\left(\sigma_{m}\right)+F_{m}{ }^{x_{L}}\left(\sigma_{m}{ }^{\tau_{m}}\right) \geq 0$, предполагая, что $\left\{\sigma_{m}^{{ }^{\tau_{m}}}, \sigma_{m}, \sigma_{m}{ }^{\pi_{m}}\right\} \subseteq P_{m}^{x_{L}}$, т.е. при предположении, что множества $Q_{m}{ }^{x_{L}}\left(\sigma_{m}{ }^{\tau_{m}}\right), Q_{m}{ }^{x_{L}}\left(\sigma_{m}\right), Q_{m}{ }^{x_{L}}\left(\sigma_{m}{ }^{\pi_{m}}\right)$ не пусты. Таким образом, для доказательства леммы достаточно при указанном предположении получить неравенство

$$
F_{m}^{x_{L}}\left(\sigma_{m}{ }^{\pi_{m}}\right)-2 F_{m}^{x_{L}}\left(\sigma_{m}\right)+F_{m}^{x_{L}}\left(\sigma_{m}{ }^{\tau_{m}}\right) \geq 0 .
$$

Поскольку множество $Q_{m}^{x_{L}}\left(\sigma_{m}\right)$ является подмножеством конечного множества, найдется такая точка $w \in Q_{m}{ }^{x_{L}}\left(\sigma_{m}\right)$, что $f(w)=F_{m}{ }^{x_{L}}\left(\sigma_{m}\right)$.

По предложению 1 леммы 3 найдутся такой индекс $j \in N$ и такая точка $v \in Q$, что

$$
v \in Q_{m}{ }^{x_{L}}\left(\sigma_{m}{ }^{\tau_{m}}\right), f(v)=F_{m}{ }^{x_{L}}\left(\sigma_{m}{ }^{\tau_{m}}\right), v_{j}=w_{j}^{\pi_{j}}, v_{m}=w_{m}{ }^{\tau_{m}}, v_{s}=w_{s}, \forall s \in N \backslash\{j, m\}, m \neq j \notin L .
$$

По предложению 2 леммы 3 найдутся такой индекс $k \in N$ и такая точка $u \in Q$, что

$$
u \in Q_{m}{ }^{x_{L}}\left(\sigma_{m}^{\pi_{m}}\right), f(u)=F_{m}{ }^{x_{L}}\left(\sigma_{m}{ }^{\pi_{m}}\right), u_{k}=w_{k}^{\tau_{k}}, u_{m}=w_{m}{ }^{\pi_{m}}, u_{s}=w_{s}, \forall s \in N \backslash\{k, m\}, m \neq k \notin L
$$

Поэтому

$$
\begin{gathered}
F_{m}{ }^{x_{L}}\left(\sigma_{m}{ }^{\tau_{m}}\right)-2 F_{m}^{x_{L}}\left(\sigma_{m}\right)+F_{m}{ }^{x_{L}}\left(\sigma_{m}^{\pi_{m}}\right)=[f(v)-f(w)]+[f(u)-f(w)]= \\
=\left[f_{j}\left(w_{j}^{\pi_{j}}\right)-f_{j}\left(w_{j}\right)+f_{m}\left(w_{m}{ }^{\tau_{m}}\right)-f_{m}\left(w_{m}\right)\right]+\left[f_{k}\left(w_{k}^{\tau_{k}}\right)-f_{k}\left(w_{k}\right)+f_{m}\left(w_{m}{ }^{\pi_{m}}\right)-f_{m}\left(w_{m}\right)\right]= \\
=\left[f_{j}\left(w_{j}^{\pi_{j}}\right)-f_{j}\left(w_{j}\right)\right]+\left[f_{k}\left(w_{k}^{\tau_{k}}\right)-f_{k}\left(w_{k}\right)\right]+\left[f_{m}\left(w_{m}^{\tau_{m}}\right)-2 f_{m}\left(w_{m}\right)+f_{m}\left(w_{m}{ }^{\pi_{m}}\right)\right] .
\end{gathered}
$$

Поскольку здесь слагаемое $\left[f_{m}\left(w_{m} \tau_{m}\right)-2 f_{m}\left(w_{m}\right)+f_{m}\left(w_{m} \pi_{m}\right)\right]$ неотрицательно по выбору функции $f(x)$, из предыдущего выражения получаем следующее неравенство

$$
F_{m}{ }^{x_{L}}\left(\sigma_{m}{ }^{\tau_{m}}\right)-2 F_{m}{ }^{x_{L}}\left(\sigma_{m}\right)+F_{m}{ }^{x_{L}}\left(\sigma_{m}{ }^{\pi_{m}}\right) \geq f_{j}\left(w_{j}{ }^{\pi_{j}}\right)-f_{j}\left(w_{j}\right)+f_{k}\left(w_{k}^{\tau_{k}}\right)-f_{k}\left(w_{k}\right) .
$$

Поскольку при $j=k$ правая часть этого неравенства неотрицательна по выбору функции $f(x)$, то в этом случае требуемое неравенство (5) и лемма очевидны. Поэтому считаем, что $j \neq k$, и с учетом этого равенства (6) и (7), представляющие по координатам точки $u$ и $v$, перепишем в следующем виде.

$$
\begin{array}{ll}
v_{j}=w_{j}{ }^{\pi_{j}}, v_{k}=w_{k}, \quad v_{m}=w_{m}{ }^{\tau_{m}}, v_{s}=w_{s}, \forall s \in N \backslash\{j, k, m\}, & j \neq k \neq m \neq j, \\
u_{j}=w_{j}, \quad u_{k}=w_{k}^{\tau_{k}}, u_{m}=w_{m}{ }^{\pi_{m}}, u_{s}=w_{s}, \forall s \in N \backslash\{j, k, m\}, & \{j, k, m\} \cap L=\varnothing .
\end{array}
$$

Из этих равенств и определений $I(v, u), I(u, v)$ последовательно получаем:

$$
\{m\}_{\subseteq}(v, u),\{j, k\} \subseteq I(u, v), I(v, u) \cap I(u, v)=\varnothing,\{m\}=I(v, u),\{j, k\}=I(u, v) .
$$

С помощью этих равенств по свойству усиленной замены множества $Q$ для различных точек $u$ и $v$, выбранных из условий (6) и (7), по индексу $j \in\{j, k\}=I(u, v)$ находим такой индекс $l \in I(v, u)=\{m\}$, что $\pi_{j}\left(\tau_{m}(u)\right)=\pi_{j}\left(\tau_{l}(u)\right) \in Q$. Полагая $y=\pi_{j}\left(\tau_{m}(u)\right)$, по лемме 2 имеем: 


$$
y_{j}=u_{j}^{\pi_{j}}, \quad y_{k}=u_{k}, y_{m}=u_{m}^{\tau_{m}}, y_{s}=u_{s}, \forall s \in N \backslash\{j, k, m\}, \quad\{j, k, m\} \cap L=\varnothing .
$$

Покажем теперь, неотрицательность правой части (8). Из (9) и (10) имеем:

$$
y_{j}=u_{j}^{\pi_{j}}=w_{j}^{\pi_{j}}, \quad y_{k}=u_{k}=w_{k}{ }^{\tau_{k}}, y_{m}=u_{m}{ }^{\tau_{m}}=w_{m}, y_{s}=u_{s}=w_{s}, \forall s \in N \backslash\{j, k, m\} .
$$

С помощью этих равенств из включения $w \in Q_{m}{ }^{x_{L}}\left(\sigma_{m}\right)$ и равенства $\{j, k, m\} \cap L=\varnothing$ следует, что $y \in Q_{m}{ }^{x_{L}}\left(\sigma_{m}\right)$. Отсюда по выбору $w$ имеем $f(y) \geq f(w)$. С помощью этого неравенства из (11) по выбору функции $f(x)$ следует $f_{j}\left(w_{j}^{\pi_{j}}\right)-f_{j}\left(w_{j}\right)+f_{k}\left(w_{k}^{\tau_{k}}\right)-f_{k}\left(w_{k}\right)=f(y)-f(w) \geq 0$. Теперь из (8) вытекает требуемое неравенство (5). Лемма доказана.

Из доказанных лемм вытекают следствия.

Дополнительные обозначения. Ниже используются следующие обозначения:

$$
\begin{gathered}
E^{\tau}=\left\{j \in N: j \notin L, j \neq m, \pi_{m}\left(\tau_{j}(x)\right) \in Q\right\}, \quad \Delta_{j}^{\tau}=f_{j}\left(x_{j}{ }^{\tau_{j}}\right)-f_{j}\left(x_{j}\right)+f_{m}\left(x_{m} \pi_{m}\right)-f_{m}\left(x_{m}\right), \\
E_{\Delta}{ }^{\tau}=\left\{j \in E^{\tau}: \Delta_{j}^{\tau}<0\right\}, \\
E^{\pi}=\left\{j \in N: j \notin L, j \neq m, \pi_{j}\left(\tau_{m}(x)\right) \in Q\right\}, \Delta_{j}{ }^{\pi}=f_{j}\left(x_{j}{ }^{\pi_{j}}\right)-f_{j}\left(x_{j}\right)+f_{m}\left(x_{m}{ }^{\tau_{m}}\right)-f_{m}\left(x_{m}\right), \\
E_{\Delta}{ }^{\pi}=\left\{j \in E^{\pi}: \Delta_{j}{ }^{\pi}<0\right\},
\end{gathered}
$$

Следствие 1. Если $x$ - решение задачи $A_{m}{ }^{x_{L}}\left(x_{m}\right)$, то $E_{\Delta}{ }^{\tau} \neq \varnothing \Rightarrow F_{m}{ }^{x_{L}}\left(x_{m}\right)>F_{m}{ }^{x_{L}}\left(x_{m}{ }^{\pi_{m}}\right)$, $E_{\Delta}{ }^{\pi} \neq \varnothing \Rightarrow F_{m}{ }^{x_{L}}\left(x_{m}\right)>F_{m}{ }^{x_{L}}\left(x_{m}{ }^{\tau_{m}}\right)$

Доказательство. Из включения $j \in E_{\Delta}{ }^{\tau}$, т.е. условий $j \in N, j \notin L, j \neq m, \pi_{m}\left(\tau_{j}(x)\right) \in Q$, $f_{j}\left(x_{j}^{\tau_{j}}\right)-f_{j}\left(x_{j}\right)+f_{m}\left(x_{m} \pi_{m}\right)-f_{m}\left(x_{m}\right)<0$, следует $f\left(\pi_{m}\left(\tau_{j}(x)\right)\right)<f(x)=F_{m}{ }^{x_{L}}\left(x_{m}\right), \quad$ и поскольку $j \notin L, m \notin L$, то $\pi_{m}\left(\tau_{j}(x)\right) \in Q_{m}{ }^{x_{L}}\left(x_{m} \pi_{m}\right)$ и потому $F_{m}{ }^{x_{L}}\left(x_{m} \pi_{m}\right) \leq f\left(\pi_{m}\left(\tau_{j}(x)\right)\right)$ и, следовательно, $F_{m}{ }^{x_{L}}\left(x_{m}{ }^{\pi_{m}}\right)<F_{m}{ }^{x_{L}}\left(x_{m}\right)$. Этим получено первое заключение следствия и так как второе - получается аналогично, следствие доказано.

Следствие 2. Если $x$ - решение задачи $A_{m}{ }^{x_{L}}\left(x_{m}\right)$, то из множеств $E_{\Delta}{ }^{\tau}, E_{\Delta}{ }^{\pi}$ непустым может быть только одно.

Доказательство. Из допущения $E_{\Delta}{ }^{\tau} \neq \varnothing \neq E_{\Delta}{ }^{\pi}$ по $F_{m}{ }^{x_{L}}\left(x_{m}{ }^{\pi_{m}}\right)-2 F_{m}{ }^{x_{L}}\left(x_{m}\right)+F_{m}{ }^{x_{L}}\left(x_{m}{ }^{\tau_{m}}\right)<0$ вопреки лемме 4. Следствие доказано.

Задача $A^{x_{L}}$.При предположениях задачи $A$ требуется минимизировать $f(x)$ на множестве $Q^{x_{L}}=\left\{\left(y_{1}, \ldots, y_{n}\right) \in Q:, i \in L \Rightarrow y_{i}=x_{i}, \forall i \in N\right\}$, т.е. найти такую точку $w \in Q^{x_{L}}, \quad$ что $f(w)=\min \left\{f(y): y \in Q^{x_{L}}\right\}$.

Следствие 3. Если $x$ - решение задачи $A_{m}{ }^{x_{L}}\left(x_{m}\right)$ и $E_{\Delta}{ }^{\tau}=\varnothing=E_{\Delta}{ }^{\pi}$, то $x$ - решение задачи $A^{x_{L}}$.

Следствие 4. Если $x$ - решение задачи $A_{m}{ }^{x_{L}}\left(x_{m}\right)$ и $E_{\Delta}{ }^{\tau}=\varnothing$, то $x$ - решение задачи минимизации $f(w)$ при условиях $w \in Q^{x_{L}}, x_{m} \prec_{m} w_{m}$.

Следствие 5. Если $x$ - решение задачи $A_{m}{ }^{x_{L}}\left(x_{m}\right)$ и $E_{\Delta}{ }^{\pi}=\varnothing$, то $x$ - решение задачи минимизации $f(w)$ при условиях $w \in Q^{x_{L}}, w_{m} \prec_{m} x_{m}$.

Замечание. С помощью лемм 3,4 и следствий 1-5 обосновывается следующий алгоритм для нахождения решения задачи $\mathbf{A}$.

Алгоритм $\boldsymbol{A}\left(\boldsymbol{x}^{0}, \varphi\right)$.В приводимом ниже описании используются: $x^{0}$ - стартовая точка множества $Q,, \varphi$ - задаваемая на множестве всех подмножеств из $N$ функция выбора из каждого подмножества его элемента. 
Ш а г 1. Положить $x=x^{0}, L=M\{\varphi(N)\}, t=2$.

Ш а г $t$.

Подшаг 0. Если $t=n+1(L=\varnothing)$, объявить $x$ искомым решением и остановиться.

В противном случае положить $m=\varphi(N)$ и заменить $L$ на $L \backslash\{m\}$; сформировать множества $E_{\Delta}^{\tau}, E_{\Delta}{ }^{\pi}$.

Если $E_{\Delta}{ }^{\tau}=\varnothing \wedge E_{\Delta}{ }^{\pi}=\varnothing$ идти на Увеличение $t$.

В противном случае:

при $E_{\Delta}{ }^{\tau} \neq \varnothing$ положить $\lambda=\tau, \mu=\pi$;

при $E_{\Delta}{ }^{\pi} \neq \varnothing$ положить $\lambda=\pi, \mu=\tau$;

положить $k=1$.

Подшаг $k$.Сформировать множество $E^{\lambda}$.

Если $E^{\lambda}=\varnothing$, идти на Увеличение $t$.

В противном случае положить $M^{\lambda}=\min \left\{4_{j}^{\lambda}: j \in E^{\lambda}\right\}$.

Если $M^{\lambda} \geq 0$, идти на Увеличение $t$.

В противном случае определить индекс $i$ из условий $i \in E^{\lambda}, \Delta_{i}^{\lambda}=M^{\lambda}$, заменить $x_{i}$ на $x_{i}^{\lambda_{i}}, x_{m}$ на $x_{m}{ }^{\mu_{m}}$,

увеличить $k$ на 1.

Увеличение $t$. Заменить $t$ на $t+1$.

Справедливо следующее утверждение.

Теорема. При предположениях задачи А алгоритм $A\left(x^{0}, \varphi\right)$ находит ее решение $x$ из любой стартовой точки $x^{0}$ при всякой функции выбора $\varphi$.

\section{БИБЛИОГРАФИЧЕСКИЙ СПИСОК}

1. Емеличев В.А., Овчинников В.Г. Симметричные суперматроиды / Докл. АН БССР, 1983. Т. 27. № 5. С. 389-391.

2. Емеличев В.А., Овчинников В.Г. К теории экстремума на координатных решетках // Докл. АН БССР. 1983. Т.27. № 7. C. 581-583.

3. Овчинников В.Г. Об одном свойстве подмножеств координатной решетки, на которых локальный и глобальный экстремумы координатно-выпуклой функции совпадают // Изв. АН БССР. Сер. Физ.-мат. науки. 1984. № 5. С. 113 / Ред. журнал «Изв. АН БССР. Сер. Физ.-мат.н.» Минск.1983. 6с.: Библ. 4 назв. Рукопись деп. в ВИНИТИ 09.01.84 № 245-84Деп.

4. Емеличев В.А., Овчинников В.Г. К теории оптимизации на антицепях, обладающих свойством замены Швейница // Кибернетика, 1985. № 2. С. 55-58.

5. Овчинников В.Г. Об одной задаче целочисленного программирования // Кибернетика, 1976. № 1. С. 131-135.

6. Пападимитриу X., Стайглии К. Комбинаторная оптимизация. Алгоритмы и сложность. М: Мир, 1985. 512c.

7. Айгнер.М. Комбинаторная теория. М.: Мир,1982. 556 с. 